\title{
L'organisation des marchés de producteurs de fruits et légumes biologiques à Nairobi, Kenya
}

\author{
Chloé Tankam* \\ AgroParisTech - centre de Clermont-Ferrand, 9, avenue Blaise-Pascal, CS 70054, 63178 Aubière cedex, France
}

\begin{abstract}
Résumé - La consommation de produits certifiés n'est plus l'apanage des pays développés. Au Kenya, les premiers marchés biologiques sont apparus à Nairobi en 2006. Ils sont approvisionnés par des maraîchers, confrontés à une diversité de défis : construire une certification biologique crédible, garantir la fraîcheur des produits et composer avec l'hétérogénéité des attentes des consommateurs. À partir de données d'enquête et du cadre analytique des coûts de transaction, nous analysons l'organisation des marchés de 2006 à 2013. Nous montrons que si la certification collective des producteurs a permis de fonder les premiers marchés, son abandon progressif n'a pas été synonyme d'effondrement des transactions. En effet, l'organisation des producteurs, qui tient d'une quasi-intégration, a permis une coordination efficace avec le marché, contribuant à la formation d'une rente que les producteurs cherchent à préserver. De plus, les relations interpersonnelles entre producteurs et consommateurs, rendues possible par la vente directe, ont contribué à la continuité des transactions malgré l'arrêt de la certification et la hausse de l'incertitude associée.
\end{abstract}

Mots clés : petit agriculteur / marché agricole domestique / agriculture biologique / coût de transaction / Kenya

\begin{abstract}
Supplying Nairobi with organic products: some insights on farmer markets' organization. Consumption of certified products is no longer the privilege of developed countries. In Kenya, the first organic markets appeared in 2006 in Nairobi. They were supplied by vegetable growers, facing different challenges: building a credible organic certification, maintaining products' freshness and dealing with the heterogeneity of consumers' expectations. Based on survey data and the transaction costs analytical framework, we analyze Kenyan organic markets between 2006 and 2013. We show that if the collective certification of producers enabled the creation of initial markets, the gradual abandonment of certification was not synonymous with the collapse of transactions. Actually, the organization of producers, which is "quasi-integrated", allowed for an efficient coordination with markets, helping to build a rent that the producers try to protect. On the other hand, interpersonal relations between producers and consumers resulting from direct sales, ensured the continuation of transactions despite the end of the certification and the related increasing uncertainty.
\end{abstract}

Keywords: smallholders / local markets / organic farming / transaction costs / Kenya

\section{Introduction}

La consommation de produits certifiés n'est plus l'apanage des pays développés. Désormais, des marchés biologiques domestiques se développent, notamment en Afrique de l'Est. Au Kenya, les premiers marchés biologiques sont apparus à Nairobi en 2006. Près de 7000 tonnes de fruits et de légumes frais y sont vendus chaque année, générant en 2012 un chiffre d'affaires de près de 2 millions d'euros, soit entre $5 \%$ et $10 \%$ du commerce de l'ensemble des fruits et légumes biologiques au Kenya. La croissance annuelle est soutenue, de l'ordre de $33 \%$ entre 2011 et 2012 (Organic Denmark, 2013).

\footnotetext{
$\bar{*}$ Auteur de correspondance $:$ chloe.tankam@agroparistech.fr
}

L'agriculture biologique est apparue au Kenya dans les années 1980. De retour d'Angleterre où ils avaient été formés aux techniques agricoles biologiques, des étudiants kenyans ont fondé une organisation (Kenyan Institute of Organic Farming) chargée de transmettre les rudiments de l'agriculture biologique aux producteurs. Progressivement, de nombreuses organisations se sont investies dans la formation des agriculteurs. L'organisation non gouvernementale (ONG) « $\mathrm{A}$ », créée en 2004, a permis de mettre en réseau ces différents acteurs.

Au début des années 2000, la multiplication d'affirmations sur la qualité - des revendications non étayées sur la qualité des processus de production - dans le secteur de l'agroalimentaire kenyan a révélé à la fois un intérêt pour des produits nouveaux et le potentiel d'une offre différenciée par un système de contrôle. 
L'ONG A a alors vu dans le développement de marchés locaux un outil de lutte contre la pauvreté pour les petits producteurs. Toutefois, elle a buté sur l'absence d'une législation sur l'agriculture biologique. Aussi a-t-elle décidé d'initier une double stratégie au niveau national :

- créer une solution de garantie : le premier organisme de certification biologique kenyan a été fondé en 2005 par un ancien membre de l'ONG A ;

- organiser l'offre : l'ONG a identifié les producteurs intéressés et a accompagné la légalisation de groupements dès 2005. Dans le même temps, elle a réalisé des formations afin de les préparer à la certification nouvellement créée. Les premiers certificats ont été délivrés entre 2006 et 2007.

En parallèle, l'ONG a poursuivi son travail de lobbying pour une reconnaissance institutionnelle de l'agriculture biologique. Elle a participé dès 2005 à la formulation d'une norme biologique est-africaine, approuvée en 2007 et progressivement mise en œuvre.

Dès 2009, la certification a été progressivement abandonnée, du fait des contraintes liées au mode de commercialisation, d'une part, et à la production certifiée, d'autre part. Toutefois, les marchés ont perduré, ce qui nous a amené à nous demander comment les producteurs ont réussi à maintenir les marchés de fruits et de légumes biologiques.

Pour répondre à cette question, nous nous sommes intéressés à la gouvernance des transactions sur les marchés de fruits et de légumes biologiques à Nairobi entre 2006, date d'apparition des premiers marchés, et 2013. Cette période est en effet marquée par l'effondrement du système de certification locale, qui a engendré de fortes incertitudes sur l'offre et la demande dans un contexte où des actifs spécifiques sont requis (Williamson, 1985). Or, en présence d'actifs spécifiques, c'està-dire d'investissements faiblement redéployables en cas de rupture de la relation entre agents, les transactions nécessitent des garanties contractuelles et organisationnelles. Pour analyser ce type de transactions qui dépassent l'échange entre acheteurs anonymes, nous mobilisons le cadre analytique de l'économie des coûts de transaction (Williamson, 1985).

Après une présentation du cadre théorique et de la méthode utilisée, nous montrerons comment les producteurs sont parvenus à construire des marchés biologiques basés sur une certification par tiers de nature privée et à maintenir les transactions malgré l'effondrement du système de certification initial.

\section{Cadre théorique}

Le caractère biologique des produits implique une asymétrie d'information supportée par les consommateurs. Ces produits font par ailleurs l'objet d'une gestion complexe en raison de leur caractère périssable et de la diversité des préférences des consommateurs. Pour ces deux raisons, les transactions nécessitent des modes de coordination que le marché classique, essentiellement fondé sur l'ajustement par les prix, ne peut prendre en charge (Brousseau et Codron, 1998).

L'économie néo-institutionnelle nous permet d'analyser des modes d'organisation autres que le seul marché. Williamson (1985) montre que le recours au marché anonyme est souvent coûteux pour les agents, mettant en lumière d'autres formes d'organisations, notamment une diversité de formes contractuelles. Le choix de l'une ou de l'autre forme s'explique par : le niveau d'incertitude, le degré de spécificité des actifs engagés et la fréquence de la transaction.

L'incertitude peut être endogène ou exogène. La première est une incertitude comportementale liée à la stratégie des acteurs. La seconde fait référence au caractère difficilement prévisible des situations dans lesquelles vont se trouver les parties. L'incertitude endogène est également liée aux difficultés de mesurer la qualité des produits (Barzel, 1982). Or, une information coûteuse à obtenir est à la base d'aléas sur les échanges de biens et de services, et en conséquence sur les arrangements institutionnels (Barzel, 1982).

La spécificité des actifs désigne le caractère peu redéployable d'un investissement (matériel ou immatériel) engagé dans la transaction. Ainsi, en cas de rupture de contrat prématurée, l'utilisation de cet actif dans une transaction alternative n'est pas envisagée sans coût ou perte de valeur productive.

L'hypothèse retenue concernant la fréquence des transactions est que plus les cocontractants ont besoin de travailler ensemble, plus la défaillance de l'un a des conséquences négatives sur l'autre.

Ici, l'incertitude exogène est liée aux aléas pesant sur la production agricole. Ces contraintes sont accentuées dans le cas de fruits et de légumes sensibles aux épisodes de stress hydrique et de petits producteurs n'ayant pas les ressources pour investir dans des outils de conservation, de transformation ou de transport. Cette incertitude est également due aux comportements des consommateurs. En effet, ceux-ci ont des goûts variés, ils montrent des préférences pour la diversité et s'appuient sur des critères de choix différents pour des produits identiques. Ainsi, des analyses portant sur le consentement à payer des consommateurs au Bénin et au Ghana pour des produits biologiques, montrent qu'ils tiennent à la fois compte de la fraîcheur, de l'absence de défaut physique ou encore de l'homogénéité dans la forme et la coloration dans leurs achats de choux et de tomates (Coulibaly et al., 2011). De plus, leurs préférences sont fluctuantes, liées à la météorologie, et donc difficiles à prévoir.

L'incertitude endogène découle des difficultés d'évaluation des caractéristiques des produits. Les caractéristiques mesurables d'un fruit (poids, couleur, teneur en sucre, etc.), accessibles au consommateur et comme on l'a vu effectivement mobilisées, restent de mauvais indicateurs de son apport nutritionnel, gustatif, ou encore de ses capacités de conservation. Le problème d'identification de la qualité est renforcé pour les produits issus de l'agriculture biologique. En effet, ce sont des biens de croyance (Darby et Karni, 1973), dont la caractéristique biologique, non identifiable même après l'achat, est très coûteuse à mesurer. Aussi est-il nécessaire d'établir un référentiel de qualité (définir un cahier des charges) et d'organiser un contrôle formel de la conformité à ce référentiel, afin d'en constituer la crédibilité. Le contrôle exante (accréditation des producteurs) et ex-post (vérification des produits) peut être réalisé par différentes formes de certification. La répétition des transactions et la réputation construite au fil du temps peuvent également décourager l'exploitation des failles des arrangements contractuels.

La spécificité des investissements est surtout liée ici au paiement du certificat biologique. Hors marchés biologiques, 
ce certificat n'a aucune valeur, car les consommateurs des marchés traditionnels ne sont pas sensibles à l'attribut biologique. À cela s'ajoute une spécificité temporelle due à la périssabilité des produits. Ainsi, en cas de défaut de clientèle sur les marchés biologiques, les producteurs sont contraints d'écouler rapidement leur production, vendue alors sur des marchés conventionnels synonymes de perte de la prime de prix.

Les étapes de définition et de garantie de la qualité génèrent des problèmes de coordination pouvant être résolus par l'intégration verticale ou par des formes hybrides. Dans ce dernier cas, plusieurs mécanismes existent face aux incertitudes intervenant avant et après la contractualisation (Ménard, 2012). La délimitation des droits de propriété, la répartition de la prise de décision, la mise en place de règles et de dispositifs d'arbitrages internes et enfin les modalités de partage de la rente varient selon que l'organisation est dotée d'un centre fort ou d'une coordination très décentralisée.

\section{Méthodologie}

La transaction que nous avons étudiée est la vente de fruits et de légumes biologiques, l'acte par lequel le producteur cède son produit contre une rémunération. L'étude de cette transaction nous amène à analyser l'organisation de la production. Pour ce faire, notre travail s'appuie sur une enquête qualitative et quantitative. La première a été menée auprès de sept acteurs de la société civile, du certificateur local et de producteurs. Au sein des deux principaux groupements approvisionnant les marchés biologiques de Nairobi, nous avons réalisé des entretiens avec huit des membres des bureaux. Les questions ont porté sur le fonctionnement des groupements et l'organisation de la vente. L'enquête quantitative repose sur un tirage aléatoire de 50 producteurs au sein de ces collectifs, composés de près de 80 individus. Afin de proposer une comparaison des prix de vente sur les marchés biologiques et conventionnels, nous introduisons un échantillon de 43 producteurs non biologiques. Il est constitué à partir d'une méthode d'appariement, basée sur les caractéristiques de l'exploitation et du ménage de notre population initiale, au sein des zones d'enquête.

\section{Résultats}

\subsection{Les producteurs des marchés biologiques de Nairobi}

En 2006, le premier marché biologique de Nairobi était un marché de producteurs. En 2013, on en dénombre cinq, dont trois marchés de producteurs, une épicerie biologique et un restaurant spécialisé. En 2013 comme en 2006, les marchés sont essentiellement approvisionnés par deux groupements de producteurs (Tab. 1). En 2013, le groupement 1 cultive en moyenne quatre espèces contre six pour le groupement 2 .

À la question des investissements réalisés pour accéder aux marchés biologiques, l'essentiel des producteurs mentionne le paiement du certificat biologique (le tarif individuel la première année s'élève à $3000 \mathrm{KES}$, soit près de 25 euros,
Tableau 1. Caractéristiques des groupements approvisionnant les marchés de producteurs biologiques de Nairobi (2013).

Table 1. Characteristics of farmer groups supplying organic markets in Nairobi (2013).

\begin{tabular}{lcc}
\hline & Groupe 1 & Groupe 2 \\
\hline $\begin{array}{l}\text { Superficie moyenne, en hectares } \\
\begin{array}{l}\text { Ancienneté de la pratique agricole, } \\
\text { en années }\end{array}\end{array}$ & 0,8 & 1,6 \\
$\begin{array}{l}\text { Ancienneté de la pratique } \\
\text { biologique, en années }\end{array}$ & 8 & 17,7 \\
$\begin{array}{l}\text { Pourcentage des producteurs } \\
\text { associant production végétale et } \\
\text { élevage }\end{array}$ & 40 & 13 \\
\hline
\end{tabular}

puis 1000 KES par an), $40 \%$ mentionnent le coût de transport pour se rendre à des sessions de formation (réalisées pour $80 \%$ des producteurs par des ONG) et $15 \%$ citent des achats de matériel agricole ou de systèmes d'irrigation. Il n'y a donc que le prix du certificat, déboursé pendant 3 à 5 ans selon les producteurs, qui corresponde à un investissement spécifique. Les producteurs équipent petit à petit les espaces de vente (avec des étals par exemple), mais la contribution d'ONG à ces dépenses ne nous permet pas de conclure à un investissement financier réel du groupement. L'enquête a permis d'évaluer les écarts de prix des produits vendus sur les marchés de producteurs biologiques et conventionnels (Tab. 2).

\subsection{Les systèmes de certification et de garantie}

En 2006, sous l'impulsion de l'ONG A, les producteurs biologiques intéressés situés à Nairobi et dans les environs ont été formés et organisés en groupements afin de mettre en place un système de contrôle interne ( $\mathrm{SCI}$ ) (Fig. 1), selon les règles de l'International Federation of Organic Agriculture Movements (IFOAM). L'entrée dans le groupement était synonyme d'abandon de la propriété de sa production, qui devenait propriété du groupement. Les producteurs élisaient un bureau chaque année, qui était chargé de contrôler les pratiques agricoles selon le référentiel défini par le certificateur ENCERT. Ce dernier était le seul habilité à délivrer le certificat biologique collectif. Son contrôle consistait à évaluer si le SCI fonctionnait bien en vérifiant la documentation interne et la qualification de l'équipe, sans procéder à un contrôle direct. Ce certificat autorisait les producteurs à vendre, à date fixe, sur ce que l'IFOAM nomme des « points de vente collectifs ", identifiés par le bureau avec l'appui de l'ONG A. Avant chaque vente, les membres du bureau communiquaient avec les producteurs par téléphone, s'informaient de l'état des récoltes et identifiaient la quantité pouvant être vendue. Il ne s'agissait pas de quotas mais d'une discussion pour convenir du panier de chacun. Les membres du bureau étaient présents sur les marchés pour contrôler la présence des producteurs.

Les années 2009 à 2010 ont constitué une période de transition entre l'arrêt progressif du paiement du certificat et la mise en place d'un système participatif de garantie (SPG) pour 
Tableau 2. Prix de détail sur les marchés de producteurs biologiques de Nairobi (septembre-novembre 2013).

Table 2. Retail prices for different products sold on organic farmer markets in Nairobi (September-November 2013).

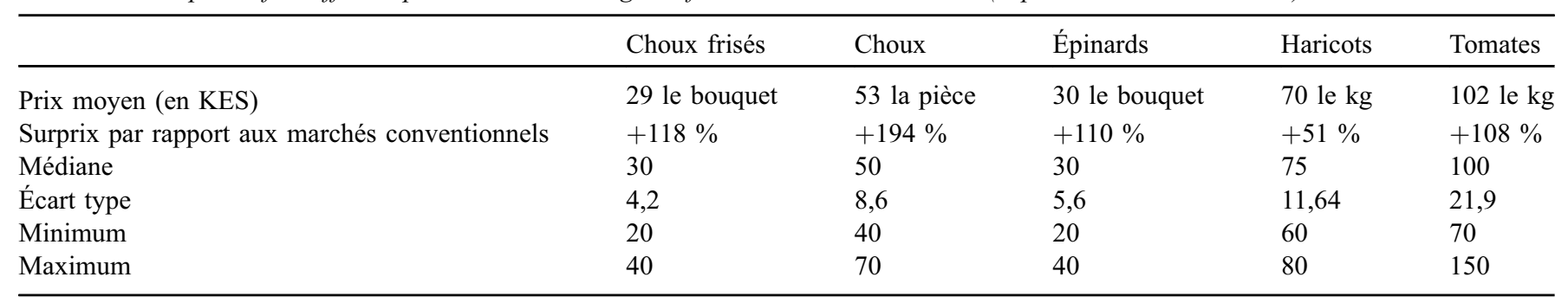

1 euro $=109$ KES (novembre 2015).

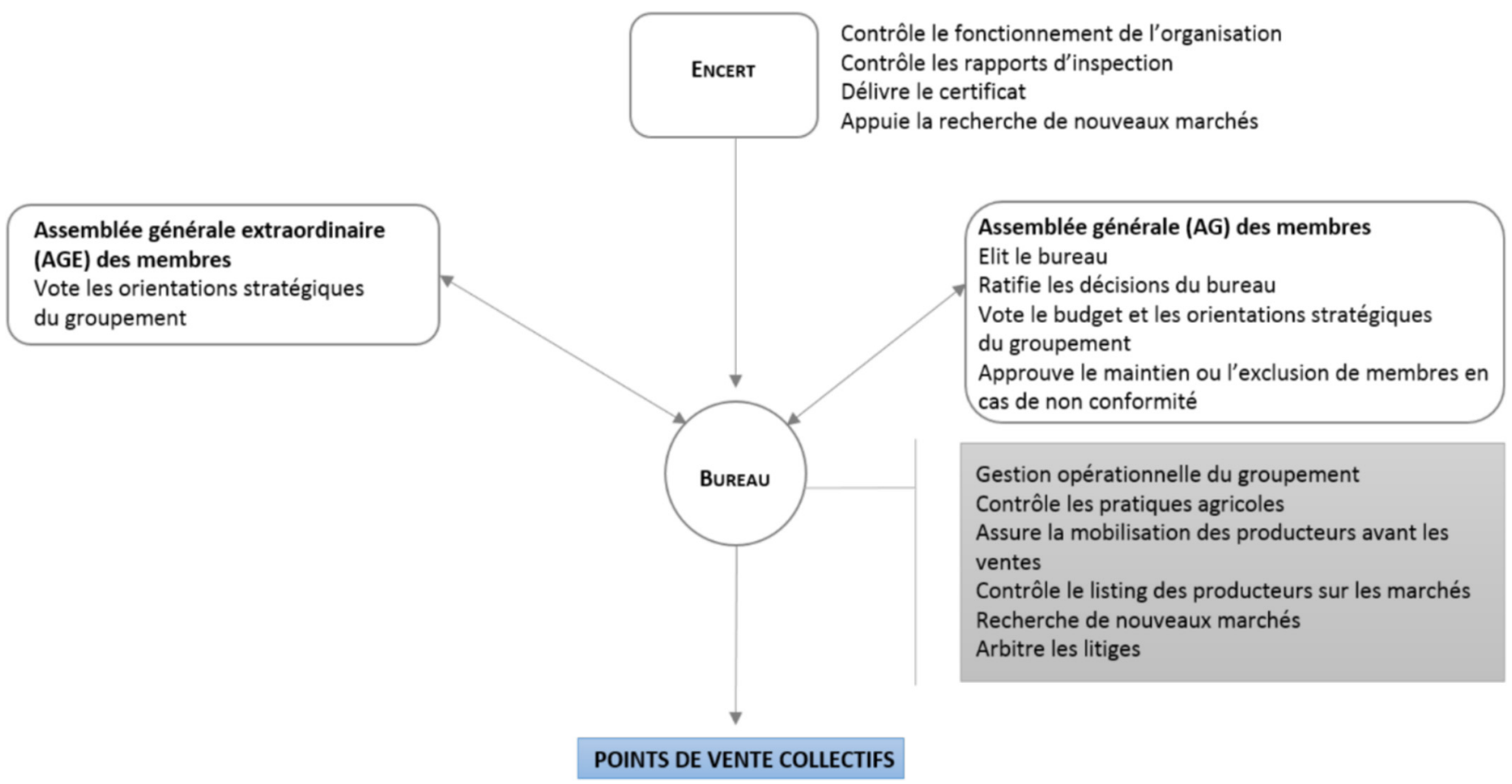

Fig. 1. Organisation du système de contrôle interne en 2006.

Fig. 1. Internal Control System's organization in 2006.

chaque groupement (Fig. 2). Ces systèmes d'assurance qualité certifient les producteurs en s'appuyant sur une participation active des acteurs concernés. Ils sont construits sur une base de confiance, de réseaux et d'échange de connaissances (May, 2008). Les SPG ont été officiellement créés en 2010. Chaque SPG peut choisir son propre cahier des charges. En l'occurrence, les deux groupements s'appuient sur la norme biologique estafricaine, permettant de bénéficier du label associé, nommé Kilimohai (Katto Andrighetto, 2013). L'ONG A, habilitée par l'IFOAM à vérifier la conformité à cette norme, en contrôle tous les 18 mois la gestion administrative et le respect du cahier des charges. Les inspections des producteurs sont réalisées tous les six mois par un comité composé de neuf membres, répartis en trois sous-groupes selon les aires géographiques. Tout producteur peut cependant décider d'y prendre part, y compris ponctuellement. Les rapports d'inspection sont transmis au comité « sanction » constitué de trois personnes : un producteur, un représentant du gouvernement et un membre d'une ONG kenyane. Les sanctions sont validées en assemblée générale.
Le SPG n'implique pas de céder la propriété de sa production au groupement. Les producteurs peuvent choisir où et quand écouler leur production, à condition de transmettre au comité " marketing » les informations sur les flux de marchandises vendues. Lui seul, en liaison avec le comité " sanction » et le bureau, est habilité à délivrer le label Kilimohai. Il reprend les activités qui étaient celles du bureau dans le cadre du SCI (inspection des marchés et contrôles sur place). Comme en 2006, les producteurs conservent le droit de fixer les prix de vente dans une certaine limite.

\subsection{L'évolution des transactions et l'émergence d'une rente organisationnelle}

Les premiers marchés biologiques de Nairobi étaient des marchés de producteurs, qui se sont maintenus malgré l'abandon de la certification. Au sein de la diversité des modes d'organisation de nature collective, le choix réalisé ici correspond à une quasi-intégration. 


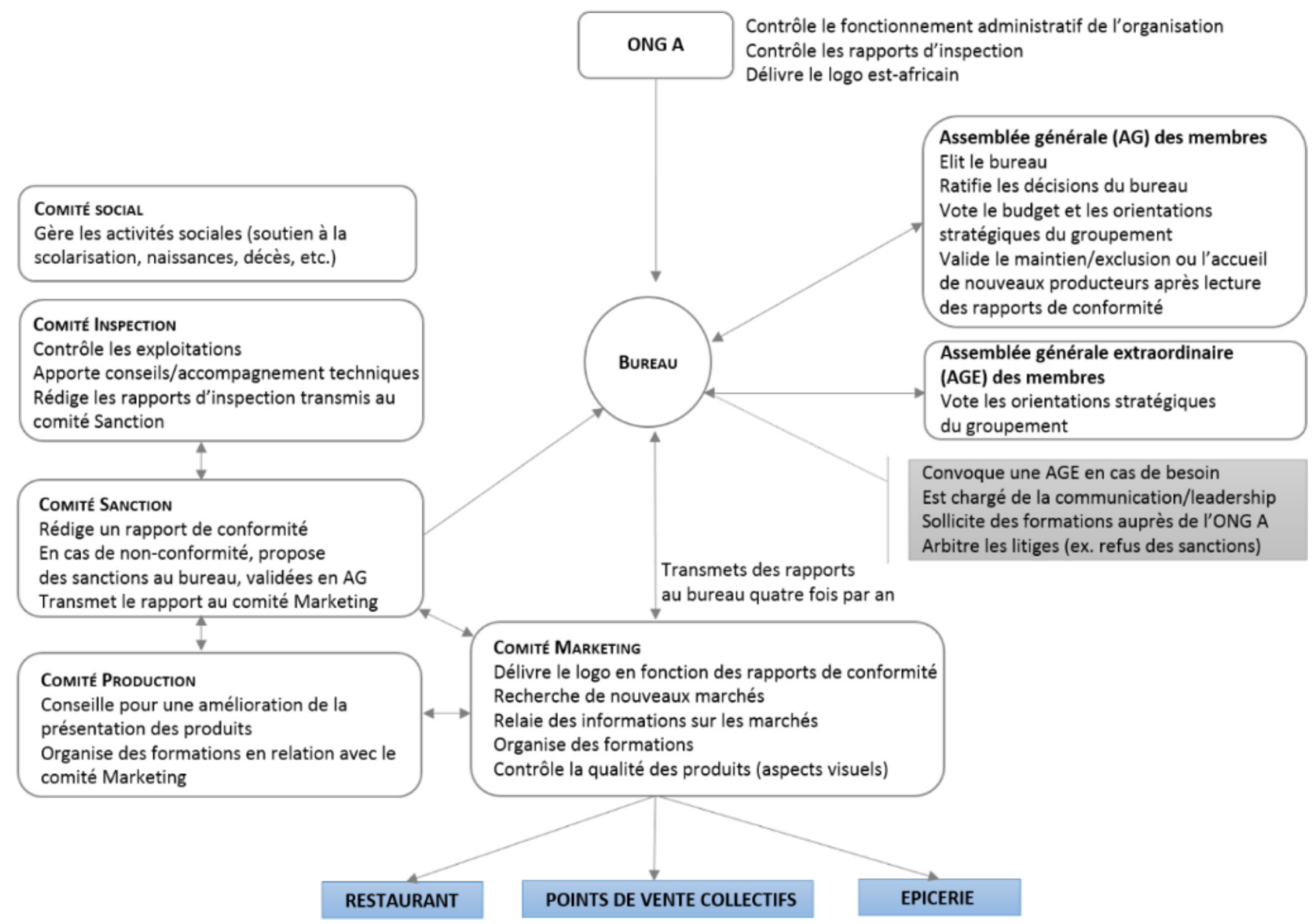

Fig. 2. Organisation d'un système participatif de garantie en 2010.

Fig. 2. Participatory Guarantee System's organization in 2010.

Les déterminants de cette forme collective résident tout d'abord dans le contexte économique et institutionnel. En effet, en 2006, face aux affirmations de qualité relevant de l'autoproclamation, la certification est apparue comme un levier de différenciation. L'absence de réglementation sur l'agriculture biologique, et donc d'un dispositif permettant de simplifier les relations contractuelles, ainsi que la production essentiellement familiale, ont contraint les acteurs à s'organiser collectivement, selon un modèle centralisé. La certification collective a permis à des petits producteurs de prendre en charge l'incertitude ex-ante et ex-post. En adhérant à une norme existante et à un dispositif de contrôle collectif, ils ont bénéficié d'économies d'échelle dans la définition du référentiel et dans la mise en œuvre du contrôle. De plus, l'organisme de certification ENCERT présentait des caractéristiques crédibles pour le consommateur. Outre son statut de tiers externe, l'entreprise utilisait différents outils pour construire sa crédibilité : son cahier des charges, inspiré de l'exemple européen, était mis en ligne, de même que les noms des producteurs. L'abandon par les producteurs de la propriété juridique de leur production permettait un transfert de pouvoirs de décision aux bureaux, qui faisaient des SCI des formes fortement intégrées. En effet, ces derniers étaient en droit de contrôler la production, d'identifier des débouchés, de vérifier la présence sur les marchés, de solliciter des quantités, ou encore d'organiser la rotation des producteurs si la demande était faible (en période d'été notamment). Le cumul des inspections et de la coordination avec le marché dotait les groupements d'une réelle capacité d'adaptation. Ces fonctions commerciales, qui demandaient un apprentissage, nécessitaient une équipe stabilisée. Cela était permis par des mandats annuels, reconduits en assemblées générales plusieurs fois. De plus, les points de vente étant des lieux privés mis à disposition essentiellement hors contrat formel, la confiance construite à la faveur du temps entre les propriétaires des lieux et les membres des bureaux jouait un rôle non négligeable.

À partir de 2009, la certification a été fragilisée par les limites du mode de commercialisation d'une part et de la production certifiée d'autre part. L'obligation d'une vente directe à date fixe a généré pour les producteurs des coûts d'opportunité, de transaction et des rigidités face à des besoins en liquidité immédiats (Tankam, 2015). De plus, la tenue de registres sur la base d'un vaste cahier des charges constituait un passage à l'écrit qui n'était pas forcément une pratique habituelle des agriculteurs. Le maintien des transactions s'avérait d'autant plus difficile que le SCI impliquait la 
complémentarité des investissements réalisés par les producteurs. En effet, la vente sur les marchés biologiques était initialement conditionnée au respect du cahier des charges et au paiement du certificat par chacun. Or, cette dépendance bilatérale renforçait les risques contractuels, en particulier le risque de holdup de la rente (Klein et al., 1978), consistant à profiter de la dépendance du cocontractant pour renégocier les termes du contrat.

Le maintien des transactions malgré cette progressive décertification s'explique par la capacité des bureaux à tenir compte des récriminations des producteurs. Ils ont introduit des zones de tolérance dans le fonctionnement des SCI, composant avec les absences des producteurs qui privilégiaient d'autres marchés. La définition collective de prix planchers a été organisée à la demande des producteurs (quelques minutes avant le début des marchés), aidant à mieux tenir compte des difficultés qu'ils rencontraient.

Les transactions ont aussi perduré parce qu'elles continuaient de trouver une clientèle. L'obligation d'avoir des points de vente collectifs et le pilotage réalisé permettaient de proposer une offre en quantité et en diversité dans des lieux identifiés pour leur accessibilité aux consommateurs. L'organisation d'un marché dans l'enceinte d'une ambassade a été la forme la plus poussée de prise en compte des coûts de transaction supportés par les consommateurs, en l'occurrence les employés de cette ambassade. La vente à date fixe aidait les producteurs à planifier les récoltes et à fournir des produits frais, autre attribut de la qualité. Dans le même temps, ces rendez-vous atténuaient le risque de lock in, c'est-à-dire l'exposition des producteurs à une décote importante de leurs produits en cas de défaillance sur les marchés biologiques (clientèle insuffisante notamment). En effet, la vente directe permettait de fidéliser une clientèle et de la sensibiliser aux contraintes de la production biologique. Les relations interpersonnelles de respect, voire d'amitié selon les dires des producteurs, ont aidé à la continuité des transactions malgré l'arrêt de la certification qui aurait pu s'accompagner d'une perte de confiance des consommateurs.

Ainsi, malgré les différents coûts mentionnés, les producteurs sont parvenus à construire une rente (Tab. 2) qui explique le maintien des transactions. Cette rente, liée au cahier des charges, est aussi de nature organisationnelle. Elle naît de la coopération des producteurs que nous venons de décrire. Son partage correspond au fait de réserver les marchés biologiques aux producteurs certifiés. Pour la conserver et faire face à la hausse des paramètres d'incertitude endogène, les bureaux ont conservé des inspections bimensuelles et leur présence sur les marchés.

Le SPG repose sur une gouvernance moins centralisée, les fonctions de l'ancien bureau du SCI étant réparties sur le groupement entre cinq comités. Il assouplit également l'intégration réalisée dans le SCI en autorisant une vente individuelle et la négociation bilatérale de prix avec l'acheteur. Cependant, le rôle du comité « marketing » est aussi de veiller à une harmonisation des prix de vente, l'infléchissement est donc relatif. L'abandon du SCI a été rendu possible par le contexte institutionnel et économique. Non seulement la norme est-africaine autorise le recours à d'autres modes de contrôle, à l'autoproclamation lorsque le producteur se déclare lui-même conforme, et autorise les SPG, mais les nouveaux acteurs économiques (épiceries et restaurants spécialisés notamment) représentent des opportunités pour les producteurs. Or, l'organisation décentralisée des SPG rend a priori possible un contrôle de flux de marchandises plus importants. Dans le même temps, en délivrant un certificat collectif, le SPG permet de conserver les investissements réalisés pour bâtir la réputation des premiers marchés.

\section{Conclusion}

L'environnement institutionnel et économique a fortement contraint le choix initial d'un signe de qualité collectif. Le contrat établi avec le certificateur a permis, en rendant publique et solennelle la volonté de coopérer, de rassurer les consommateurs et donc de fonder le marché. Si, en conditionnant la vente à la certification le SCI initie des risques contractuels, il permet cependant, par une coordination efficace avec le marché, l'émergence d'une rente organisationnelle qui incite les producteurs à maintenir des mécanismes de contrôle. Cette rente, et la vente directe, expliquent le maintien des marchés malgré la hausse de l'incertitude endogène.

Si nous pouvons expliquer ce qui a conduit aux SPG, ceuxci méritent d'être mieux caractérisés. En effet, le manque de données ne permet pas d'en évaluer l'efficacité dans la coordination avec les marchés. Deux points nous semblent mériter complément :

- évaluer les coûts des SPG et les coûts d'opportunité liés au pilotage déconcentré.

- caractériser la place qui y est donnée à l'apprentissage et à la formation des producteurs.

En effet, il ressort que c'est un des facteurs qui a permis aux SCI de se maintenir. Le développement de produits nouveaux va donc nécessiter, en Afrique notamment, des recherches approfondies sur le rôle de la gestion des apprentissages dans la gouvernance des transactions.

\section{Références}

Barzel Y. 1982. Measurement cost and the organization of markets. Journal of Law and Economics 25: 27-48.

Brousseau E, Codron J. 1998. La complémentarité entre formes de gouvernance (le cas de l'approvisionnement des grandes surfaces en fruits de contre-saison). Économie Rurale 245: 75-83.

Coulibaly O, Nouhoheflin T, Aitchedji C, Cherry A, Adegbola P. 2011. Consumers' perceptions and willingness to pay for organically grown vegetables. International Journal of Vegetable Science 17: 349-362.

Darby MR, Karni E. 1973. Free competition and the optimal amount of fraud. Journal of Law and Economics 16: 67-88.

Katto Andrighetto J. 2013. Participatory guarantee systems in East Africa case studies from Kenya, Tanzania and Uganda. Bonn: International Federation of Organic Agriculture Movements (IFOAM).

Klein B, Crawford RG, Alchian AA. 1978. Vertical integration, appropriable rents, and the competitive contracting process. Journal of Law and Economics xxi: 297-326.

May C. 2008. Petit guide des SPG ou comment développer et faire fonctionner les systèmes participatifs de garantie. Technical report, IFOAM. Disponible sur https://shop.ifoam.bio/fr/system/files/ products/downloadable_products/pgs_guidelines_fr_web.pdf. 
Ménard C. 2012. Hybrid modes of organization. Alliances, joint ventures, networks, and other 'strange'animals. The Handbook of Organizational Economics. Princeton: Princeton University Press. Organic Denmark. 2013. Enhancing the Coordination of Organic products access to Markets in East Africa (ECOMEA). Available at http://okologi.dk/media/504852/ecomea-project-document.pdf.
Tankam C. 2015. Analyse économique des nouveaux marchés biologiques : le cas des marchés biologiques domestiques kenyans. Thèse, Université d'Auvergne Clermont-Ferrand 1. Disponible sur https://hal.archives-ouvertes.fr/tel-01488967/document.

Williamson OE. 1985. The economic institutions of capitalism. New York: Free Press.

Citation de l'article : Tankam C. 2017. L'organisation des marchés de producteurs de fruits et légumes biologiques à Nairobi, Kenya. Cah. Agric. 26: 35006 